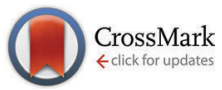

Cite this: Phys. Chem. Chem. Phys., 2015, 17, 21198

Received 3rd November 2014 Accepted 22nd December 2014

DOI: 10.1039/c4cp05087h

www.rsc.org/pccp

\section{Surface- and tip-enhanced resonant Raman scattering from CdSe nanocrystals}

\author{
E. Sheremet, $\dagger^{\star a}$ A. G. Milekhin, ${ }^{\text {bc }}$ R. D. Rodriguez, $\ddagger^{a}$ T. Weiss, ${ }^{d}$ M. Nesterov, ${ }^{d}$ \\ E. E. Rodyakina, ${ }^{\text {bc }}$ O. D. Gordan, ${ }^{a}$ L. L. Sveshnikova, ${ }^{b}$ T. A. Duda, ${ }^{b}$ V. A. Gridchin, ${ }^{e}$ \\ V. M. Dzhagan, ${ }^{a}$ M. Hietschold ${ }^{f}$ and D. R. T. Zahn $\ddagger^{a}$
}

\begin{abstract}
Surface- and tip-enhanced resonant Raman scattering (resonant SERS and TERS) by optical phonons in a monolayer of CdSe quantum dots (QDS) is demonstrated. The SERS enhancement was achieved by employing plasmonically active substrates consisting of gold arrays with varying nanocluster diameters prepared by electron-beam lithography. The magnitude of the SERS enhancement depends on the localized surface plasmon resonance (LSPR) energy, which is determined by the structural parameters. The LSPR positions as a function of nanocluster diameter were experimentally determined from spectroscopic micro-ellipsometry, and compared to numerical simulations showing good qualitative agreement. The monolayer of CdSe QDs was deposited by the Langmuir-Blodgett-based technique on the SERS substrates. By tuning the excitation energy close to the band gap of the CdSe QDs and to the LSPR energy, resonant SERS by longitudinal optical (LO) phonons of CdSe QDs was realized. A SERS enhancement factor of $2 \times 10^{3}$ was achieved. This allowed the detection of higher order LO modes of CdSe QDs, evidencing the high crystalline quality of QDs. The dependence of LO phonon mode intensity on the size of $\mathrm{Au}$ nanoclusters reveals a resonant character, suggesting that the electromagnetic mechanism of the SERS enhancement is dominant. Finally, the resonant TERS spectrum from CdSe QDs was obtained using electrochemically etched gold tips providing an enhancement on the order of $10^{4}$. This is an important step towards the detection of the phonon spectrum from a single QD.
\end{abstract}

\section{Introduction}

Quantum dots (QDs) attracted intense research interest due to their potential for electronic and optoelectronic applications. ${ }^{1}$ Among them, CdSe QDs are promising for numerous applications in photodetectors, field-effect transistors, solar cells, light-emitting diodes. $^{2-4}$ The information about the phonon spectrum of QDs is important for practical applications, since the phonons determine the channels for charge carrier relaxation in QD based devices. ${ }^{5-7}$ Phonon spectra from an ensemble of QDs can be obtained by

\footnotetext{
${ }^{a}$ Semiconductor Physics, Technische Universität Chemnitz, D-09107 Chemnitz, Germany.E-mail: evgeniya.sheremet@physik.tu-chemnitz.de

${ }^{b}$ A.V. Rzhanov Institute of Semiconductor Physics, pr. Lavrentieva, 13, Novosibirsk630090, Russia. E-mail: amilekhin@hotmail.com

${ }^{c}$ Novosibirsk State University, Pirogov str. 2, Novosibirsk 630090, Russia

${ }^{d} 4$ th Physics Institute and Research Center SCoPE, University of Stuttgart, D-70550 Stuttgart, Germany

${ }^{e}$ Novosibirsk State Technical University, K. Marx av.20, Novosibirsk 630073, Russia

${ }^{f}$ Solid Surfaces Analysis Group, Technische Universität Chemnitz,

D-09107 Chemnitz, Germany

$\dagger$ Current address: Solid Surfaces Analysis Group, Technische Universität Chemnitz, D-09107 Chemnitz, Germany.

\# Member of Cluster of Excellence "Center for Advancing Electronics Dresden" cfAED.
}

Raman spectroscopy (RS), which provides information on QD size, ${ }^{8}$ shape, ${ }^{9}$ as well as strain relaxation ${ }^{10}$ and atomic intermixing. ${ }^{11,12}$ Since the properties of QDs are strongly sensitive to the QD size and environment, analyzing the phonon spectrum of a single QD would give valuable insight into the individual QD properties. However, the intensity of the Raman signal from a single QD is often too low to be detected, and therefore methods to amplify it are required.

One of the well-established methods to enhance the Raman signal is resonant Raman spectroscopy (RRS), which is achieved by choosing the excitation wavelength close to (or at) the band gap energy of the QD system studied. However, enhancement provided by RRS reaches $10^{3}$, and this is not sufficient for the detection of very small amounts of material, such as a single QD.

Surface- and tip-enhanced Raman spectroscopies (SERS and TERS) are two actively developing approaches, which drastically improve the sensitivity and spatial resolution of RS. They are based on the enhancement of the electromagnetic field by localized surface plasmons (LSPs) of metallic nanoclusters to drastically increase the intensity of relatively weak Raman scattering by up to $10^{7}$ times. $^{13,14}$ In the case of organic molecules, single-molecule sensitivity was achieved by both SERS $^{15,16}$ and TERS. ${ }^{17-19}$ These methods were also employed 
to detect the signal from low amounts of inorganic materials, such as $\mathrm{CdS}$, $\mathrm{CdSe}, \mathrm{GaN}, \mathrm{Cu}_{x} \mathrm{~S}$, and $\mathrm{ZnO}^{20-24}$ Recently, resonant SERS was successfully employed to analyze the anisotropy of the phonon spectrum of a single CdSe nanoplatelet. ${ }^{25}$ The scattering volume of a single QD is 30 times smaller than that of a nanoplatelet, which makes the task of detecting a single QD challenging.

TERS is the ultimate case of SERS, it breaks the diffraction limit of light and thus drastically improves the spatial resolution of the conventional RS. This is accomplished using the apex of a tip as a single plasmonic scatterer, and moving it in a controlled manner with respect to the sample. The power of TERS to improve the spatial resolution was demonstrated on single nanowires and crystallites ${ }^{26-28}$ with a resolution comparable to that of AFM imaging. Raman and photoluminescence spectra of CdSe nanowires were investigated as well, achieving optical resolution below AFM resolution in the same experiment due to the square-law field dependence of both the excitation and the emission rates. ${ }^{29,30}$ The improved spatial resolution of TERS also allowed mapping of stress and composition within a single quantum dot. ${ }^{31}$

The enhancement of the Raman signal can be maximized by simultaneously employing RRS and SERS (or TERS). This requires matching the excitation energy (wavelength) to the band gap of QDs, as well as to the localized surface plasmon resonance (LSPR) energy of a plasmonic structure. The LSPR position can be tuned by changing the size, the shape, and the distance between the plasmonic nanostructures. ${ }^{32}$ Electron beam lithography provides unique control of all those parameters allowing optimization of the SERS signal. ${ }^{33,34}$

In this paper, both resonant SERS and TERS were employed in order to detect a monolayer of CdSe QDs deposited by the Langmuir-Blodgett-based technique.

\section{Results and discussion}

SERS substrates are prepared on Si substrates, and consist of gold nanocluster arrays with the period of 150 , while the nanocluster diameters were systematically varied from $70 \mathrm{~nm}$ to $140 \mathrm{~nm}$. It was shown that the arrays of gold nanoclusters serve as effective SERS substrates providing SERS by organic and biological substances, ${ }^{35-37}$ including our previous studies using the CoPc dye as a Raman probe. ${ }^{38,39}$ In our case the CdSe QDs of $5.2 \mathrm{~nm}$ size deposited by the Langmuir-Blodgett-based technique play the role of the Raman probe. The deposition procedure is described in the experimental section. The QDs form a homogeneous coverage on the $\mathrm{Si}$ substrate as well as on the gold nanocluster arrays, as confirmed by SEM measurements (Fig. 1).

\section{LSPR position}

The LSPR positions were characterized by spectroscopic microellipsometry. The LSPR position was determined as the maximum of the extinction coefficient spectrum extracted from ellipsometry measurements. The dependence of the LSPR position on the gold nanocluster diameter is shown in Fig. 2. The data show an increase of the LSPR position with an increase in the gold nanocluster

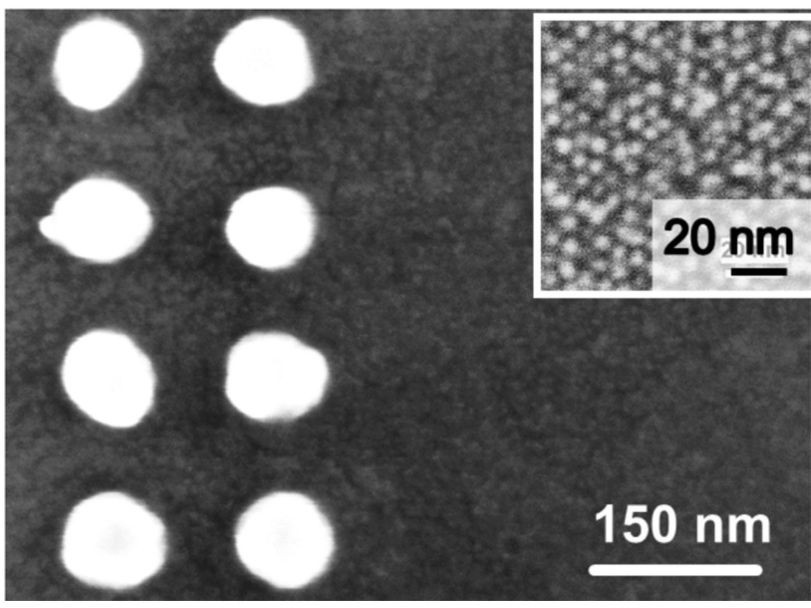

Fig. 1 SEM image of an edge of the nanostructured gold array (left side, white areas are the gold nanoclusters) demonstrating homogeneous coverage of both nanostructure and a Si substrate with a monolayer of CdSe QDs. Inset shows the high resolution SEM image of the CdSe QD monolayer on the Si substrate.

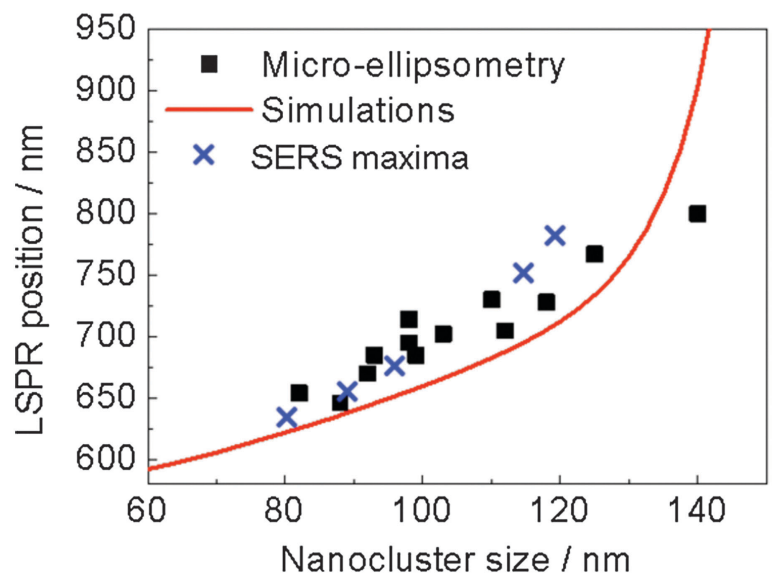

Fig. 2 LSPR position determined from ellipsometry measurements (squares) and simulations (solid line) depending on gold nanocluster size for the $150 \mathrm{~nm}$ array period. The crosses indicate the wavelengths, for which maxima of the SERS enhancement were observed (see section surface-enhanced Raman spectroscopy of CdSe QDs).

diameter. These results are in agreement with the well-established behaviour of the LSPR position. ${ }^{32}$

To better understand the resonant behaviour of the structure, the extinction coefficients and LSPR positions were numerically modeled using the Fourier modal method with adaptive spatial resolution and matched coordinates. ${ }^{40,41}$ It is important to note that the LSPR position strongly depends on the dielectric function of the gold nanocluster environment. Direct experimental determination of the dielectric function around the gold nanoclusters is challenging, since the optical response is dominated by the signal of the gold nanoclusters. Therefore, the optical response of the silicon substrate covered by a monolayer of CdSe QDs was measured. The dielectric function of the CdSe QD monolayer can be approximated for this thickness range by a Cauchy dispersion. As a result, in the spectral region of interest, 
the optical response of the system is equivalent to a $11 \mathrm{~nm} \mathrm{SiO}_{2}$ layer on the Si substrate. The calculated LSPR positions of the gold nanoclusters on the $\mathrm{Si}$ substrate with $11 \mathrm{~nm} \mathrm{SiO}{ }_{2}$ thickness give good agreement with the experiment (Fig. 2), showing the red shift of the LSPR position with an increase in the nanocluster diameter. When the distance between the neighbouring nanoclusters drops below $20 \mathrm{~nm}$, the near-field coupling effect leads to further red shift of the LSPR position.

\section{Surface-enhanced Raman spectroscopy of CdSe QDS}

The Raman and SERS spectra of the CdSe monolayer are shown in Fig. 3. In this case, Raman spectra are resonant, since the excitation wavelength $(632.8 \mathrm{~nm})$ approaches the specified emission maximum $(610 \mathrm{~nm})$ determined by the energy of interband transition in CdSe QDs. The Raman spectra of the CdSe QDs deposited on a Si substrate show only clear bands of the Si substrate (second-order transverse acoustic (2TA) phonon mode at $300 \mathrm{~cm}^{-1}$ and LO mode at $520 \mathrm{~cm}^{-1}$ ) and no detectable signal of the CdSe QDs. The resonant SERS allows the detection of an intense first order Raman scattering by LO phonons of the CdSe QDs at $207 \mathrm{~cm}^{-1}$, which is in agreement with previously reported values, ${ }^{42-44}$ as well as of higher order modes. For the specified CdSe size of $5.2 \mathrm{~nm}$ no strong phonon confinement effect and therefore no confinement induced shift of LO phonon mode in CdSe QDs are expected, as shown experimentally ${ }^{44}$ and theoretically. ${ }^{45}$ The observation of higher order features in the Raman spectrum reveals a remarkable crystalline quality of the QDs. The first order feature also exhibits a broad background, which can be attributed either to the enhanced signal from amorphous Se on the QD surface, ${ }^{46}$ or to scattering from the SERS substrate. The effective SERS enhancement factor (EF) was estimated as a ratio of the LO signal intensity of CdSe QDs on the SERS substrate to the noise level on the Si substrate, since the CdSe signal was not detectable on the Si substrate. The resulting EF is approximately $2 \times 10^{3}$.

The normalized dependence of the LO phonon mode intensity on the $\mathrm{Au}$ nanocluster diameter obtained using different excitation wavelengths is shown in Fig. 4. The curves show a clear resonant behaviour, with the maximum of the Raman

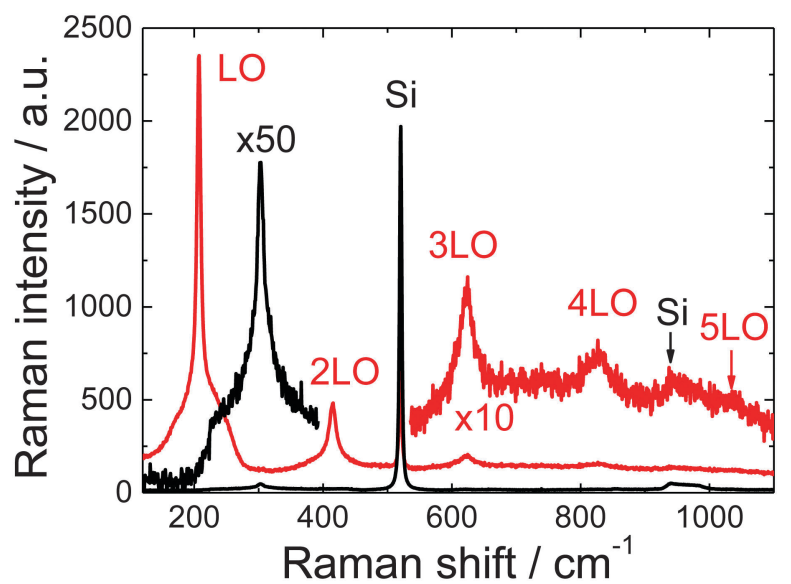

Fig. 3 Raman (black curve) and SERS (red curve) spectra of CdSe QDs measured with a $632.8 \mathrm{~nm}$ laser line for a nanocluster diameter of $80 \mathrm{~nm}$.

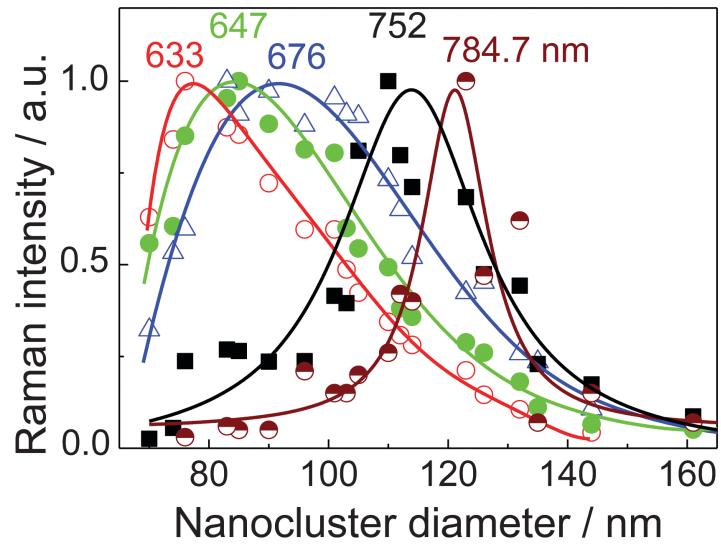

Fig. 4 Normalized dependence of the Raman intensity of the CdSe LO phonon on the gold nanocluster diameter for different wavelengths (indicated in the figure).

signal shifting to larger nanocluster sizes when increasing the excitation wavelength. The nanocluster sizes and wavelengths, for which the maximum SERS enhancement was observed, were compared to the LSPR positions measured with micro-ellipsometry (crosses in Fig. 2). Clearly, for a given nanocluster size, the maximum enhancement is observed when the excitation wavelength matches the measured maximum LSPR position. This implies that the resulting SERS enhancement is dominated by the electromagnetic enhancement, rather than the intrinsic resonance of CdSe NCs or chemical enhancement.

\section{TERS measurements}

Detection of fewer QDs as compared to SERS measurements was achieved in a TERS experiment on the CdSe QD monolayer deposited on the SERS structure with $88 \mathrm{~nm}$ nanocluster diameter, which is close to the resonance observed for the $632.8 \mathrm{~nm}$ excitation line according to Fig. 4. Note that the shift of the resonance can be caused by the metallic tip when it approaches an Au nanocluster. The comparison of SERS and TERS spectra is shown in Fig. 5. In the TERS experiment, the area of the laser spot is approximately $1.5 \times$ $2.5 \mu^{2}$, giving rise to the so-called far-field signal (tip up in Fig. $5 \mathrm{~b}$ ). When the electrochemically etched gold tip was brought into contact with the QDs, the Raman signal from the QDs located directly under the tip apex was enhanced (tip down in Fig. 5b). It was possible to achieve a 100\% increase of the CdSe LO mode intensity at $207 \mathrm{~cm}^{-1}$. Similar to SERS spectra, the increased background at low frequency can be attributed to enhancement of the signal from amorphous Se on the QD surface, and partially to elastic scattering from the tip.

The latter could lead to an increase of the Raman signal not related to the near-field enhancement. ${ }^{47,48}$ However, since the intensity of the Si peak remains the same, such a signal increase can be excluded, ${ }^{39}$ and the TERS EF can be estimated using the formula:

$$
\mathrm{EF}=\frac{I_{\text {down }}-I_{\text {up }}}{I_{\text {up }}} \cdot \frac{V_{\text {ill }}}{V_{\text {enh }}}
$$

where $I_{\text {down }}\left(I_{\text {up }}\right)$ - intensity of the CdSe Raman signal with the tip down (up); $V_{\text {ill }}\left(V_{\text {enh }}\right)$ - is the illuminated (enhanced) volume. 

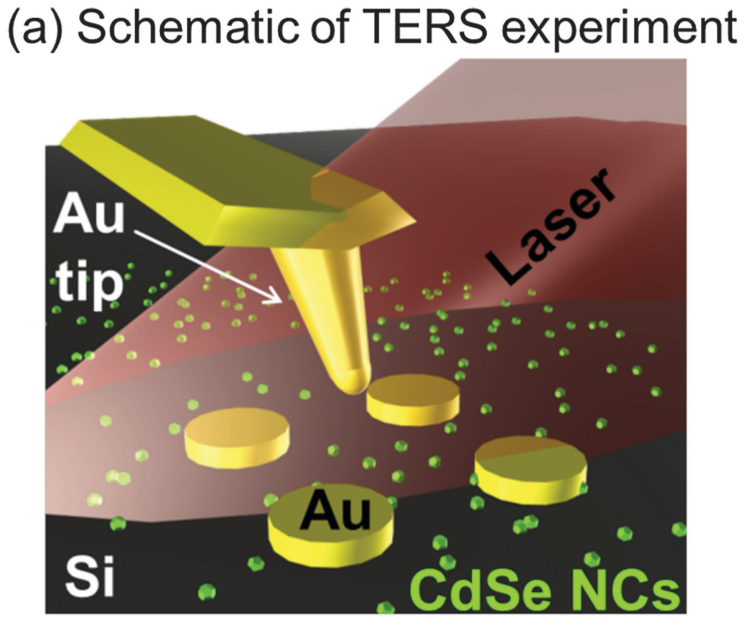

(b) TERS spectra

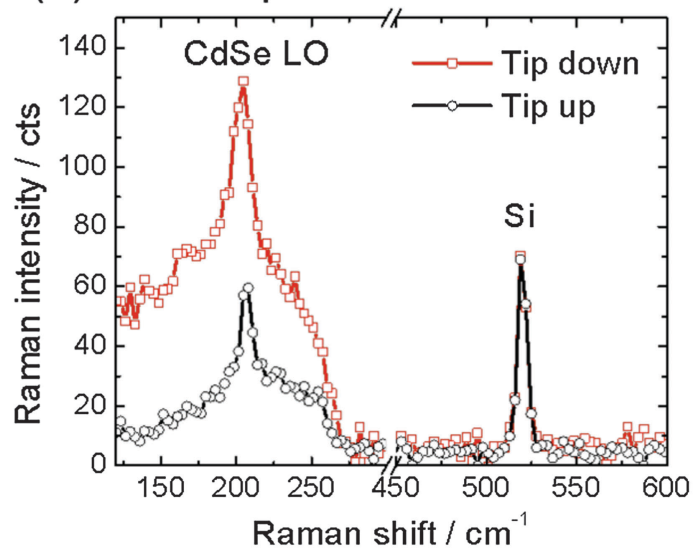

Fig. 5 TERS of the CdSe monolayer on the SERS substrate. (a) Schematic of the experiment (not in scale). (b) Raman spectra of tip in contact (tip down) and tip away from the sample (tip up).

Assuming that the CdSe film is thin enough to neglect the drop of field enhancement within the film, we normalize the Raman intensities by area instead of volume. Notice that this assumption leads to an underestimation of the enhancement factor, in order to have a conservative estimation.

The area of the illuminated volume is defined by the laser spot size. Then the enhanced area diameter was estimated to be ${ }^{49}$

$$
d=\sqrt{R_{\text {tip }} g}
$$

where $R_{\text {tip }}$ is the tip radius (typically below $50 \mathrm{~nm}$ among the tips prepared in our lab as confirmed by SEM), and $g$ - the gap between the tip and the substrate defined by the QD size. The TERS EF was estimated to be $6 \times 10^{4} \pm 14 \%$.

Solving the problem of packing smaller circles in a larger circle, we can then expect seven CdSe QDs in the enhanced area.

\section{Experimental}

\section{SERS substrate preparation}

The $10 \times 10 \mu \mathrm{m}^{2}$ gold nanocluster arrays with a period of $150 \mathrm{~nm}$ to be used as SERS substrates were prepared by a direct-write electron beam lithography process. The electron resist (polymethyl methacrylate $950 \mathrm{~K}$ ) deposited on an $\mathrm{Si}(100)$ substrate was structured with an electron beam (20 kV, Raith-150, Germany), and the patterned resist was covered with a $5 \mathrm{~nm}$ thick film of Ti and a $40 \mathrm{~nm}$ thick film of Au. Finally, the remaining resist was removed by a lift-off process in dimethylformamide to obtain isolated gold nanoclusters with diameters from $70 \mathrm{~nm}$ to $140 \mathrm{~nm}$.

\section{Langmuir-Blodgett-based deposition}

The deposition of CdSe NCs was performed using the LangmuirBlodgett-based (LB-based) technique. Pre-synthesized CdSe NCs of $5.2 \mathrm{~nm}$ diameter (Lumidot ${ }^{\mathrm{TM}}$ 610) were mixed with behenic acid and deposited using the LB-based approach. Note that it is not clear where exactly NCs are incorporated in organics. After 20 cycles of LB-based deposition, the sample was annealed at $200{ }^{\circ} \mathrm{C}$ in order to remove the organic matrix, and a monolayer of CdSe NCs was formed. The removal of organic layer was confirmed by infrared (IR) and energy-dispersive X-ray (EDX) spectroscopies.

\section{Micro-ellipsometry}

The SERS substrates covered by a monolayer of CdSe QDs were investigated by nulling micro-ellipsometry to determine the LSP resonance position using a Spectroscopic Imaging Ellipsometer (EP3-SE, Accurion $\mathrm{GmbH}$ ) with the spectral range of 365-1000 nm (3.4-1.24 eV).

The measured sample was modelled as a multi-layer system, taking into account the optical properties of the substrate. Here a Maxwell-Garnet effective medium approximation (MG-EMA) was used. The dielectric functions of silicon, silicon dioxide and gold were taken from the commercially available Woollam WVASE32 3.6 database. The substrate was modelled as a silicon substrate with a silicon oxide thickness of $11 \mathrm{~nm}$. The dielectric function obtained for the MG-EMA layer is strongly dominated by the metallic nanostructures on top of the substrate, matching the effective dielectric function of the system. In turn this makes the determination of the LSPR peak possible even by directly recalculating measured effective optical constants to the extinction coefficient.

\section{Simulations}

The extinction coefficients and LSP positions were numerically modeled using the Fourier modal method with adaptive spatial resolution and matched coordinates. ${ }^{40,41}$ This approach is very efficient for calculating layered periodic systems fabricated by electron beam lithography. The model volume consists of a semi-infinite silicon substrate (constant refractive index of $n=3.68)$, a $11 \mathrm{~nm} \mathrm{SiO}$ layer $(n=1.46)$ and a $40 \mathrm{~nm}$ high gold disk. The analytical model of the dielectric function for gold was taken from the work of Etchegoin et al. ${ }^{50}$

\section{Micro-Raman measurements}

Raman experiments were carried out using Jobin Yvon Dilor XY800, Horiba T64000, and Labram HR800 spectrometers equipped with optical microscopes (the laser beam was focused to a spot with a size of approximately $1 \mu \mathrm{m}^{2}$ using $100 \times$ objective with N.A. 0.9) in a backscattering geometry at room temperature. The $632.8 \mathrm{~nm}(1.96 \mathrm{eV})$ line of a HeNe laser (Siemens), and 
several lines of a $\mathrm{Kr}^{+}$laser (Coherent) were used as excitation sources. A laser power below $1 \mathrm{~mW}$ was used in the experiment to avoid possible effects of local heating. For the SERS spectra (on Au nanocluster arrays) $10 \mathrm{~s}$ acquisition time, 15 accumulations were performed; each Raman spectrum (on $\mathrm{Si}$ ) was acquired for $30 \mathrm{~s}, 60$ accumulations, and the Raman intensity was divided by a factor of 3 in order to compare the intensities.

\section{TERS measurements}

For the TERS experiments, a Raman spectrometer (LabRam HR800) was coupled to an Agilent 5420 (Agilent, USA) atomic force microscope (AFM). A custom-made optical extension delivered the laser light to the AFM tip at an angle of $70^{\circ}$ with respect to the sample normal axis. A $50 \times$ magnification long-working-distance objective lens with N.A.0.42 (Mitutoyo, Japan) was used both to focus the laser light onto the tip in the vicinity of the sample surface and to collect the scattered light. The $632.8 \mathrm{~nm}$ laser line with a laser power of $0.4 \mathrm{~mW}$ on the sample was used. The mirror and objective lens of the optical extension unit were driven by piezoelectric motors to improve the system stability when aligning the laser on the tip. A fully metallic gold tip cantilever was used in contact mode in the AFM for the TERS measurements. The cantilever preparation procedure is described elsewhere. ${ }^{51}$ The tip apex was sharpened by electrochemical etching. The TERS spectra were recorded with tip down (in contact with the surface) and tip up (tip retracted by $1 \mu \mathrm{m}$ ) on the gold nanocluster array with a $150 \mathrm{~nm}$ period. The gold nanocluster diameter was $88 \mathrm{~nm}$. The TERS spectra were accumulated for $5 \mathrm{~s}$.

\section{Conclusions}

The LSPR positions of gold nanocluster arrays fabricated by electron beam lithography were measured using spectroscopic micro-ellipsometry and compared to values calculated using the Fourier modal method. Both the experimental and the computational approach show that the LSPR position red-shifts with an increase in the nanocluster diameter. Using these SERS substrates, an enhancement factor as high as $2 \times 10^{3}$ for the Raman scattering by the CdSe LO phonon mode was demonstrated for a CdSe QD monolayer. SERS allowed the detection of higher order phonon modes for CdSe QDs, demonstrating their remarkable crystalline quality. The dependence of SERS enhancement on the nanocluster diameter for different excitation wavelengths reveals a resonance behaviour suggesting that the electromagnetic enhancement mechanism dominates. Finally, TERS results obtained from the CdSe QD monolayer on the SERS substrate allowed acquiring the spectrum of fewer QDs as compared to micro-Raman measurements with an additional enhancement on the order of $10^{4}$. This opens up a prospective for studying phonon spectra of QD arrays of a low areal density.

\section{Acknowledgements}

Authors thank DFG project ZA146/22-1, DFG Research Unit 1713 SMINT, Russian Science Foundation (project 14-12-01037), and
Cluster of Excellence for Advanced Electronics Dresden (cfaed) for financial support. AM thanks DAAD for providing a scholarship. This work was performed in the context of the European COST Action MP1302 Nanospectroscopy. M. N. acknowledges financial support through an Alexander von Humboldt research fellowship.

\section{Notes and references}

1 T. Trindade, P. O'Brien and N. L. Pickett, Chem. Mater., 2001, 13, 3843-3858.

2 L. J. Zhao, L. F. Hu and X. S. Fang, Adv. Funct. Mater., 2012, 22, 1551-1566.

3 V. I. Klimov, A. A. Mikhailovsky, S. Xu, A. Malko, J. A. Hollingsworth, C. A. Leatherdale, H. J. Eisler and M. G. Bawendi, Science, 2000, 290, 314-317.

4 R. C. Somers, M. G. Bawendi and D. G. Nocera, Chem. Soc. Rev., 2007, 36, 579-591.

5 A. V. Uskov, A. P. Jauho, B. Tromborg, J. Mork and R. Lang, Phys. Rev. Lett., 2000, 85, 1516-1519.

6 A. J. Nozik, Annu. Rev. Phys. Chem., 2001, 52, 193-231.

7 V. I. Klimov, D. W. McBranch, C. A. Leatherdale and M. G. Bawendi, Phys. Rev. B: Condens. Matter Mater. Phys., 1999, 60, 13740-13749.

8 D. A. Tenne, A. K. Bakarov, A. I. Toropov and D. R. T. Zahn, Phys. E, 2002, 13, 199-202.

9 D. A. Tenne, A. K. Bakarov, A. I. Toropov and D. R. T. Zahn, Phys. E, 2002, 13, 199-202.

10 J. Ibanez, A. Patane, M. Henini, L. Eaves, S. Hernandez, R. Cusco, L. Artus, Y. G. Musikhin and P. N. Brounkov, Appl. Phys. Lett., 2003, 83, 3069-3071.

11 J. Ibanez, R. Cusco, L. Artus, M. Henini, A. Patane and L. Eaves, Appl. Phys. Lett., 2006, 88, 141905.

12 A. Milekhin, A. Toropov, A. Kalagin and D. R. T. Zahn, Chin. J. Phys., 2011, 49, 71-76.

13 D. Cialla, A. Maerz, R. Boehme, F. Theil, K. Weber, M. Schmitt and J. Popp, Anal. Bioanal. Chem., 2012, 403, 27-54.

14 J. Stadler, T. Schmid and R. Zenobi, Nanoscale, 2012, 4, 1856-1870.

15 S. M. Nie and S. R. Emery, Science, 1997, 275, 1102-1106.

16 K. Kneipp, Y. Wang, H. Kneipp, L. T. Perelman, I. Itzkan, R. Dasari and M. S. Feld, Phys. Rev. Lett., 1997, 78, 1667-1670.

17 R. Zhang, Y. Zhang, Z. C. Dong, S. Jiang, C. Zhang, L. G. Chen, L. Zhang, Y. Liao, J. Aizpurua, Y. Luo, J. L. Yang and J. G. Hou, Nature, 2013, 498, 82-86.

18 W. Zhang, B. S. Yeo, T. Schmid and R. Zenobi, J. Phys. Chem. C, 2007, 111, 1733-1738.

19 M. D. Sonntag, J. M. Klingsporn, L. K. Garibay, J. M. Roberts, J. A. Dieringer, T. Seideman, K. A. Scheidt, L. Jensen, G. C. Schatz and R. P. Van Duyne, J. Phys. Chem. C, 2012, 116, 478-483.

20 J. T. Hugall, J. J. Baumberg and S. Mahajan, Appl. Phys. Lett., 2009, 95, 141111.

21 A. G. Milekhin, L. L. Sveshnikova, T. A. Duda, N. V. Surovtsev, S. V. Adichtchev and D. R. T. Zahn, JETP Lett., 2008, 88, 799-801. 
22 A. G. Milekhin, N. A. Yeryukov, L. L. Sveshnikova, T. A. Duda, S. S. Kosolobov, A. V. Latyshev, N. V. Surovtsev, S. V. Adichtchev, C. Himcinschi, E. I. Zenkevich, W.-B. Jian and D. R. T. Zahn, J. Phys. Chem. C, 2012, 116, 17164-17168.

23 A. G. Milekhin, N. A. Yeryukov, L. L. Sveshnikova, T. A. Duda, E. I. Zenkevich, S. S. Kosolobov, A. V. Latyshev, C. Himcinski, N. V. Surovtsev, S. V. Adichtchev, Z. C. Feng, C. C. Wu, D. S. Wuu and D. R. T. Zahn, J. Exp. Theor. Phys, 2011, 113, 983-991.

24 N. A. Yeryukov, A. G. Milekhin, L. L. Sveshnikova, T. A. Duda, L. D. Pokrovsky, A. K. Gutakovskii, S. A. Batsanov, E. E. Rodyakina, A. V. Latyshev and D. R. T. Zahn, J. Phys. Chem. C, 2014, 118, 23409-23414.

25 D. O. Sigle, J. T. Hugall, S. Ithurria, B. Dubertret and J. J. Baumberg, Phys. Rev. Lett., 2014, 113, 087402.

26 N. Marquestaut, D. Talaga, L. Servant, P. Yang, P. Pauzauskie and F. Lagugne-Labarthet, J. Raman Spectrosc., 2009, 40, 1441-1445.

27 N. Kazemi-Zanjani, E. Kergrene, L. Liu, T.-K. Sham and F. Lagugne-Labarthet, Sensors, 2013, 13, 12744-12759.

28 Y. Ogawa, Y. Yuasa, F. Minami and S. Oda, Appl. Phys. Lett., 2011, 99, 053112.

29 M. Boehmler and A. Hartschuh, ChemPhysChem, 2012, 13, 927-929.

30 M. Boehmler, Z. Wang, A. Myalitsin, A. Mews and A. Hartschuh, Angew. Chem., Int. Ed., 2011, 50, 11536-11538.

31 Y. Ogawa, T. Toizumi, F. Minami and A. V. Baranov, Phys. Rev. B: Condens. Matter Mater. Phys., 2011, 83, 081302(R).

32 W. Kiefer and S. Schlücker, Surface enhanced Raman spectroscopy: analytical, biophysical and life science applications, John Wiley \& Sons, 2013.

33 M. K. Fan, G. F. S. Andrade and A. G. Brolo, Anal. Chim. Acta, 2011, 693, 7-25.

34 D. Cialla, A. Marz, R. Bohme, F. Theil, K. Weber, M. Schmitt and J. Popp, Anal. Bioanal. Chem., 2012, 403, 27-54.

35 M. Kahl, E. Voges, S. Kostrewa, C. Viets and W. Hill, Sens. Actuators, B, 1998, 51, 285-291.

36 C. J. Choi, Z. Xu, H.-Y. Wu, G. L. Liu and B. T. Cunningham, Nanotechnology, 2010, 21, 415301.
37 R. Stosch, F. Yaghobian, T. Weimann, R. J. C. Brown, M. J. T. Milton and B. Guettler, Nanotechnology, 2011, 22, 105303.

38 A. G. Milekhin, N. A. Yeryukov, L. L. Sveshnikova, T. A. Duda, E. E. Rodyakina, E. S. Sheremet, M. Ludemann, O. D. Gordan, A. V. Latyshev and D. R. T. Zahn, Thin Solid Films, 2013, 543, 35-40.

39 E. Sheremet, R. D. Rodriguez, D. R. T. Zahn, A. G. Milekhin, E. E. Rodyakina and A. V. Latyshev, J. Vac. Sci. Technol., B: Nanotechnol. Microelectron.: Mater., Process., Meas., Phenom., 2014, 32, 04E110.

40 T. Weiss, N. A. Gippius, S. G. Tikhodeev, G. Granet and H. Giessen, J. Opt. Soc. Am. A, 2011, 28, 238-244.

41 T. Weiss, G. Granet, N. A. Gippius, S. G. Tikhodeev and H. Giessen, Opt. Express, 2009, 17, 8051-8061.

42 S. K. Islam, M. A. Sohel and J. R. Lombardi, J. Phys. Chem. C, 2014, 118, 19415-19421.

43 B. K. Rai, H. D. Bist, R. S. Katiyar, M. T. S. Nair, P. K. Nair and A. Mannivannan, J. Appl. Phys., 1997, 82, 1310-1319.

44 Y. N. Hwang, S. H. Park and D. Kim, Phys. Rev. B: Condens. Matter Mater. Phys., 1999, 59, 7285-7288.

45 C. Trallero-Giner, A. Debernardi, M. Cardona, E. MenendezProupin and A. I. Ekimov, Phys. Rev. B: Condens. Matter Mater. Phys., 1998, 57, 4664-4669.

46 A. A. Baganich, V. I. Mikla, D. G. Semak, A. P. Sokolov and A. P. Shebanin, Phys. Status Solidi B, 1991, 166, 297-302.

47 D. Roy, C. M. Williams and K. Mingard, J. Vac. Sci. Technol., B: Nanotechnol. Microelectron.: Mater., Process., Meas., Phenom., 2010, 28, 631-634.

48 N. Kumar, A. Rae and D. Roy, Appl. Phys. Lett., 2014, 104, 123106.

49 I. Romero, J. Aizpurua, G. W. Bryant and F. J. Garcia de Abajo, Opt. Express, 2006, 14, 9988-9999.

50 P. G. Etchegoin, E. C. Le Ru and M. Meyer, J. Chem. Phys., 2006, 125, 164705.

51 R. D. Rodriguez, E. Sheremet, S. Mueller, O. D. Gordan, A. Villabona, S. Schulze, M. Hietschold and D. R. T. Zahn, Rev. Sci. Instrum., 2012, 83, 123708. 